\title{
Four-dimensional $N=1$ theories, S-fold constraints on T-branes, and behaviors in IR and UV
}

\author{
Yusuke Kimura \\ KEK Theory Center, Institute of Particle and Nuclear Studies, KEK, \\ 1-1 Oho, Tsukuba, Ibaraki 305-0801, Japan \\ E-mail: kimurayu@post.kek.jp
}

AbstraCt: We analyze four-dimensional (4d) $N=1$ superconformal field theories (SCFTs) obtained as deformations of $4 \mathrm{~d} N=2 \mathrm{SCFTs}$ on S-folds by tilting 7-branes. Geometric compatibility with the structures of S-folds constrains the forms of T-branes. As a result, brane monodromies are constrained. We also discuss two $4 \mathrm{~d} N=1$ theories on probe D3-branes, where the two theories behave identically in IR, but they originate from different theories in UV. Studying the global structure of their geometry is useful in constructing these two theories.

Keywords: Field Theories in Higher Dimensions, Supersymmetric Gauge Theory

ArXiv EPRINT: 2011.04460 


\section{Contents}

1 Introduction

2 Brane monodromies in $4 \mathrm{~d} N=1$ theories as deformations of $4 \mathrm{~d} N=2$ SCFTs on S-folds

2.1 Reviews of $4 \mathrm{~d} N=3$ theory and $4 \mathrm{~d} N=2$ SCFTs on S-folds

$2.24 \mathrm{~d} N=1$ SCFTs as deformations of $4 \mathrm{~d} N=2$ SCFTs on S-folds, T-branes in $4 \mathrm{~d} N=1 \mathrm{~S}$-fold and brane monodromy

3 Two $4 \mathrm{~d} N=1$ theories behaving identically in IR but behaving differently in UV

4 Puzzle in the global structure of the gauge group

\section{Introduction}

There have been numerous advances in four-dimensional (4d) $N=2$ supersymmetric gauge theories. Seiberg and Witten obtained the exact prepotential for low-energy effective theories $[1,2]$, which is among the major advances in this field. There have been vigorous efforts in the field since this progress; for example, [3-23].

4d $N=2$ superconformal field theories (SCFTs) have recently been studied by utilizing S-fold constructions [16, 17]. A few years before these studies, the authors of [24] constructed $4 \mathrm{~d}$ theories with $N=3$ supersymmetry $^{1}$ by considering finite cyclic groups $\mathbb{Z}_{k}$ acting on torus fibrations, wherein the $\mathbb{Z}_{k}$-actions also act on torus fibers. The $4 \mathrm{~d}$ $N=2$ SCFTs studied in [17] flow to $4 \mathrm{~d} N=3$ theories, as constructed in [24], by mass deformations.

The subjects of study in this note are $4 \mathrm{~d} N=1 \mathrm{SCFTs}$ on S-folds ${ }^{2}$ obtained as in the context of deforming the $4 \mathrm{~d} N=3$ theories constructed in [24]. It would be natural to consider deforming the $4 \mathrm{~d} N=2$ SCFTs in [17] to yield such superconformal field theories, and we take this approach to study $4 \mathrm{~d} N=1$ SCFTs.

There are several advantages to analyzing $4 \mathrm{~d} N=1$ SCFTs in this manner. For example, the resulting $4 \mathrm{~d} N=1$ SCFTs relate to $4 \mathrm{~d} N=2$ and $N=3$ theories by construction, and they are also relevant to studies of the structures of S-folds. Furthermore,

\footnotetext{
${ }^{1}$ Properties of $4 \mathrm{~d} N=3$ theories were discussed in [25]. Discussions of $4 \mathrm{~d} N=3$ theories can also be found in [16, 26-34].

${ }^{2}$ See also [16] for a discussion of $N=1$ S-fold.
} 
as we discuss later in this note, they are also related to T-branes [35]. ${ }^{3}$ Recent progress of T-branes can be found, e.g., in [37-44].

We utilize the operation of tilting 7-branes in $4 \mathrm{~d} N=2$ SCFTs to yield $4 \mathrm{~d} N=1$ SCFTs, as described in $[45,46]$. This operation corresponds to allowing the Higgs field $\phi$ on the 7-branes stack to have a non-zero position-dependent vacuum expectation value (vev) over the base space, and as a result a correction term is added to the superpotential [45]. Deformation generated by this operation provides an opportunity to consider T-branes when $\phi$ and $\phi^{\dagger}$ do not commute [35].

In the $4 \mathrm{~d}$ F-theory background with a gauge field flux, $\phi$ and $\phi^{\dagger}$ do not need to commute [45]. When they do not commute, the $N=2$ supersymmetry is broken to $N=1[45,46]$. We study the $4 \mathrm{~d} N=1$ SCFT on the D3-brane probing a stack of 7-branes. A D3-brane probes ${ }^{4}$ the infrared (IR) $4 \mathrm{~d} N=1 \mathrm{SCFT}$, which corresponds to seeing a local patch of a global geometry.

There are two themes of this note. The first is to analyze $4 \mathrm{~d} N=1$ theories $^{5}$ obtained as deformations of $4 \mathrm{~d} N=3$ theories, as aforementioned. In the situation where $\phi$ and $\phi^{\dagger}$ do not commute, there is room to consider the effect of T-branes. Because the geometry considered here is an S-fold built as the quotient of elliptic fibration with finite cyclic group $\mathbb{Z}_{k}$ actions, the equations describing T-branes are constrained to be compatible with the $\mathbb{Z}_{k}$ actions.

A T-brane is given by a spectral equation $[57,58]$ that yields a multifold cover of a stack of 7 -branes, where the 7-brane stack is given by $z=0$. Because the parameter $z$ transverse to the stack of 7 -branes now has an equivalence relation

$$
z \sim e^{\frac{2 \pi i}{k}} z
$$

under the $\mathbb{Z}_{k}$ action, a consistency condition is imposed on the spectral equation of T-branes to make them compatible with the equivalence relation (1.1). A physical consequence of this compatibility argument is that brane monodromies [57, 59-61] are constrained owing to the quotient action of the $\mathbb{Z}_{k}$ groups.

The other theme is to discuss the physical phenomenon where two $4 \mathrm{~d} N=1$ SCFTs in the IR limit are indistinguishable but behave quite differently in the ultraviolet (UV) limit. We utilize a global perspective of geometry in F-theory to observe this phenomenon. This phenomenon occurs because the two globally distinct geometries have locally identical structures in a local patch that a D3-brane probes, making them appear the same in IR, but their difference becomes evident in UV.

The S-fold constructions discussed in [17, 24] were obtained as $\mathbb{Z}_{k}$-actions on elliptic fibrations over an open base wherein the complex structures of the elliptic fibers are fixed over the entire base, so the complex structures of the total spaces are compatible with the $\mathbb{Z}_{k}$ actions. However, elliptic fibrations can be constructed over a closed base space wherein the torus fibers have constant fixed complex structures over the base. Some nontrivial examples of such global geometries can be found, such as those in [62-67]. Here, global

\footnotetext{
${ }^{3}$ Nilpotent Higgs fields on branes were discussed in [36].

${ }^{4}$ Studies of D3-branes probing F-theory singularities can be found, for example, in [47-51].

${ }^{5}$ For recent studies on $4 \mathrm{~d} N=1$ theories, see, for example, [16, 52-56].
} 
geometries represent elliptic fibrations over compact base spaces. ${ }^{6}$ Such global geometries have some applications in $4 \mathrm{~d} N=1$ theories. We can observe a physical phenomenon that might be interesting: one can construct two global geometries on which physics appear completely identical in the IR limit, but in the UV limit, they describe different physical theories. This is owing to the fact that while the two geometries have different structures globally, their geometric structures appear locally identical. Physically, in the IR limit, they yield two identical $4 \mathrm{~d} N=1 \mathrm{SCFTs}$ probed by D3-branes that flow to very different theories in UV.

In recent years, there has been progress in the appearance of discrete gauge groups in F-theory, e.g., in [63-65, 68-81]. The compactification spaces in F-theory, wherein a discrete gauge group arises, are genus-one fibrations that "lack a global section" [69]. By tuning the coefficients as parameters of the moduli of such geometries without a global section, one can deform the geometry to an elliptic fibration with global sections. In the moduli space, physics on a genus-one fibration lacking a global section and physics on an elliptic fibration with global sections are very different; a discrete gauge symmetry arises in the former geometry, while a discrete gauge symmetry does not form and U(1) does form in the latter geometry. If we consider inserting a D3-brane probe into this geometric deformation, the characteristic physical phenomenon that we mentioned previously can be observed: the D3-brane is insensitive to the subtle deformation in geometry from genusone fibration without a global section into an elliptic fibration with sections. When one moves to UV, the effect of the global structure of the geometry becomes explicit, and the corresponding physical theories before and after the deformation behave very differently. Our argument in section 3 suggested that a U(1) factor coming from the global sections of the elliptic fibration and a discrete gauge group arising from the geometry of the genus-one fibration lacking a global section are not reflected in the flavor symmetry group of the $4 \mathrm{~d}$ theory on the probe D3-brane.

As a byproduct of the discussion of the second theme, we observe a puzzle in $4 \mathrm{~d}$ SCFT concerning a subtlety in the structure of the flavor symmetry group. An elliptic fibration has the notion of a "global section." This can be seen as a copy of the base space embedded in the total elliptic fibration [82]. If an elliptic fibration admits a global section, global sections form an Abelian group known as a "Mordell-Weil group." This group decomposes into a direct sum of free part $\mathbb{Z}^{r}$ and torsion part $\prod_{i} \mathbb{Z}_{i}$. In F-theory compactification [82-84], ${ }^{7}$ the structure of the Mordell-Weil group is reflected in the gauge group structure; the rank of the Mordell-Weil group ${ }^{8}$ yields the number of U(1)s formed in F-theory [82], and the global structure of the gauge group is divided by the Mordell-Weil torsion [111-113]. ${ }^{9}$ Is the structure of such Mordell-Weil torsion dividing the flavor symmetry group observed in "local" 4d SCFTs probed by D3-branes? Two opposing answers to this question seem

\footnotetext{
${ }^{6}$ For example, an elliptic fibration over $\mathbb{C}^{3}$ is a "local" geometry because $\mathbb{C}^{3}$ is not closed; in contrast, as a base, an elliptic fibration over $\mathbb{P}^{3}$ describes a global geometry because $\mathbb{P}^{3}$ is closed.

${ }^{7}$ Local models of F-theory model constructions are discussed in [85-88]. Studies of the compactification geometry of F-theory from the global perspective can be found, e.g., in [89-110].

${ }^{8}$ The rank of the Mordell-Weil group is the number of copies of $\mathbb{Z}$ in the free part.

${ }^{9}$ Computations of the Mordell-Weil torsions of elliptic fibrations in F-theory compactifications and the global structures of the gauge groups that form on the 7-branes are also discussed in [64-67, 78].
} 
possible, as we will demonstrate. Because the two views are opposite, one must choose one of the possible two viewpoints. We discuss this puzzle in section 4 .

By gauge group in sections 3 and 4, we mean the gauge group of spacetime when the theory is compactified on a Calabi-Yau 4-fold. In the D3-brane probe theory, this symmetry is perceived as (part of) the flavor symmetry of the $4 \mathrm{~d}$ theory.

We would like to make a remark that, when we discuss UV theories in this study, they come from the D3-branes probing compact genus-one fibered Calabi-Yau 4-folds, and 4d gravity is not entirely decoupled. The flow from UV theory to IR theory is the decoupling limit. When we consider a limit at which the size of the Calabi-Yau space becomes large enough, however, the theory approaches the situation where the D3-brane probes a noncompact space and the gravity decouples. Considering this limit if necessary, we do not consider the effect of gravity in this note.

When gravity is not decoupled, because the 7-branes are wrapped on a compact space, the gauge field on the 7-branes is dynamical. Thus, the symmetries on the 7-branes yield the gauge groups in UV theories. Since the gauge field is decoupled in the decoupling limit, in the D3-brane probe theory in the IR the symmetry on the 7-branes is perceived as the flavor symmetry of the $4 \mathrm{~d}$ theory.

In section 2.1, we briefly review the $4 \mathrm{~d} N=3$ theories constructed in [24] and the $4 \mathrm{~d}$ $N=2$ SCFTs realized in F-theory on S-folds in [17] as deformations of $4 \mathrm{~d} N=3$ theories. We construct and analyze $4 \mathrm{~d} N=1 \mathrm{SCFTs}$ as deformations of these theories in section 2.2. Tilting 7-branes deforms $4 \mathrm{~d} N=2$ SCFTs, which yields $4 \mathrm{~d} N=1$ SCFTs in IR [46]. After we discuss this construction, we analyze brane monodromies in the resulting $4 \mathrm{~d} N=1$ theories in section 2.2. The structures of T-branes are constrained by the symmetry of S-fold constructions. We analyze the constraints that brane monodromies receive as a consequence of this.

We discuss in section 3 two $4 \mathrm{~d} N=1$ theories that behave in an identical manner in IR but flow to very distinct theories in UV. In these constructions, considering the global aspects of the geometry is essential. When a similar argument is applied to the global structure of the gauge group formed on 7-branes, we observe a puzzle, which we discuss in section 4 . We present our concluding remarks in section 5 alongside some open problems.

\section{Brane monodromies in $4 \mathrm{~d} N=1$ theories as deformations of $4 \mathrm{~d} N=2$ SCFTs on S-folds}

\subsection{Reviews of $4 \mathrm{~d} N=3$ theory and $4 \mathrm{~d} N=2$ SCFTs on S-folds}

We first briefly review the $4 \mathrm{~d} N=3$ theories constructed in [24]. We then review the $4 \mathrm{~d}$ $N=2$ SCFTs on S-folds constructed in [17]. We will discuss $4 \mathrm{~d} N=1 \mathrm{SCFTs}$ obtained as deformations of these theories, and T-branes and brane monodromies in the resulting $4 \mathrm{~d}$ $N=1$ theories in section 2.2. We review the $4 \mathrm{~d} N=2$ and $N=3$ theories in this section, as we require them to construct $4 \mathrm{~d} N=1$ theories.

F-theory compactification on the product $\mathbb{C}^{3} \times T^{2}$ yields a $4 \mathrm{~d} N=4$ theory. The coordinates of $\mathbb{C}^{3}$ are denoted by $z_{1}, z_{2}$, and $z_{3}$, and the complex coordinate of the twotorus in the product is denoted by $z_{4}$. The authors of [24] considered the following action 
of a finite cyclic group $\mathbb{Z}_{k}$ on the product $\mathbb{C}^{3} \times T^{2}$ :

$$
\begin{aligned}
& z_{1} \rightarrow e^{\frac{2 \pi i}{k}} z_{1} \\
& z_{2} \rightarrow e^{-\frac{2 \pi i}{k}} z_{2} \\
& z_{3} \rightarrow e^{\frac{2 \pi i}{k}} z_{3} \\
& z_{4} \rightarrow e^{-\frac{2 \pi i}{k}} z_{4} .
\end{aligned}
$$

Here, the complex structure $\tau$ of the two-torus as an elliptic fiber is constant over the base $\mathbb{C}^{3}$, so the product $\mathbb{C}^{3} \times T^{2}$ is a direct product as a complex manifold. Because the $\mathbb{Z}_{k}$-action acts on the two-torus as a fiber, it is necessary to confirm that the action is compatible with the elliptic fibration structure of $\mathbb{C}^{3} \times T^{2}$. It was shown in [24] that the compatibility conditions require the order $k$ of the cyclic group $\mathbb{Z}_{k}$ to take one of the values $k=2,3,4$, or 6 , and the complex structure of the elliptic curve as a fiber must take a specific value for each of the values of $k$ [24].

We describe the complex structures of elliptic fibers of an elliptic fibration in detail here. An elliptic curve (two-torus seen as a complex curve) is described by a Weierstrass equation. A Weierstrass equation is an equation of the following form:

$$
y^{2}=x^{3}+f x+g .
$$

Then, the complex structure of an elliptic curve as a fiber of the fibration is uniquely labeled by a j-invariant, which is an invariant under the isomorphisms of elliptic curves. A j-invariant $j$ is given in terms of the Weierstrass coefficients $f, g$, as follows [114]:

$$
j=\frac{1728 \cdot 4 f^{3}}{4 f^{3}+27 g^{2}} .
$$

Modular parameter $\tau$ also specifies the complex structure of an elliptic curve. $\tau$ takes a value in $\mathbb{C}$ modulo $\mathrm{SL}(2, \mathbb{Z})$-action.

When the order $k$ of the group $\mathbb{Z}_{k}$ takes the values $k=3,4,6, N=4$ supersymmetry breaks down to $N=3$, and as a result of the orbifold action (2.1), a $4 \mathrm{~d} N=3$ theory is obtained [24].

Before we review $4 \mathrm{~d} N=2 \mathrm{SCFTs}$ on S-folds, we make a remark. Construction of a genus-one fibration whose elliptic fiber has a constant complex structure throughout the base, that is not a direct product of a two-torus as a fiber and the base space, is possible. Genus-one fibered K3 surfaces, whose fibers have constant complex structures $\tau=\exp \left(\frac{\pi i}{3}\right)$ and $\tau=i$ over the base $\mathbb{P}^{1}$ (equivalently, they have j-invariants 0 and 1728 , respectively) can be found in $[63,64] .{ }^{10}$ These global geometries play a role in the construction of $4 \mathrm{~d}$ $N=1$ theories in section 3 .

The authors of [17] constructed $4 \mathrm{~d} N=2$ SCFTs by considering F-theory compactifications on S-folds as deformations of the $4 \mathrm{~d} N=3$ theories constructed in [24], and we briefly review this construction.

\footnotetext{
${ }^{10}$ Higher dimensional generalizations to genus-one fibered Calabi-Yau 3-folds and genus-one fibered Calabi-Yau 4-folds can be found in [65-67].
} 
The S-folds in [17] were built by acting finite cyclic groups $\mathbb{Z}_{k}$ on the space $K 3^{\circ} \times \mathbb{C}^{2}$. Here, $K 3^{\circ}$ denotes an open patch in an elliptic K3 surface such that the base of $K 3^{\circ}$ is $\mathbb{C}$. $K 3^{\circ}$ is an elliptic fibration over $\mathbb{C}$. The coordinate of this base $\mathbb{C}$ is denoted as $z_{1}$, and the coordinates of $\mathbb{C}^{2}$ in the product $K 3^{\circ} \times \mathbb{C}^{2}$ are denoted as $z_{2}, z_{3} . z_{1}$ yields the coordinate transverse to the stacks of 7 -branes, and $z_{2}, z_{3}$ yield the coordinates parallel to the stacks of 7-branes. $z_{1}$ is identified with a chiral superfield, $Z_{1}$, which parameterizes the Coulomb branch, and $z_{2}, z_{3}$ are identified with a decoupled hypermultiplet, $Z_{2}, Z_{3}$ in the $4 \mathrm{~d}$ theory on the probe D3-brane. The $\mathbb{Z}_{k}$-action acting on $K 3^{\circ} \times \mathbb{C}^{2}$ is relaxed from condition (2.1) to the following condition [17]:

$$
\begin{aligned}
& z_{1} \rightarrow e^{\frac{2 \pi i}{k}} z_{1} \\
& z_{2} \rightarrow e^{i \phi_{2}} z_{2} \\
& z_{3} \rightarrow e^{i \phi_{3}} z_{3},
\end{aligned}
$$

where $\phi_{2}$ and $\phi_{3}$ are required to satisfy

$$
\phi_{2}+\phi_{3}=0 \bmod 2 \pi n \text {. }
$$

This construction yields S-folds, and F-theory on the resulting S-folds with a probe D3brane yields $4 \mathrm{~d} N=2$ SCFTs [17].

Analogous to $4 \mathrm{~d} N=3$ theories, compatibility conditions are imposed on the values of $k$ and the complex structures of the elliptic fibers. The complex structure of the fibers of the elliptic fibration $K 3^{\circ}$ must be constant over the base $\mathbb{C}$, i.e., the axio-dilaton must take a constant value over $\mathbb{C}^{3} . \Delta_{7}$ is used to represent the scaling dimension of the Coulomb branch $(\mathrm{CB})$ operator for the 7-brane in $K 3^{\circ} \times \mathbb{C}^{2}$. When $\mathbb{Z}_{k}$-groups act on the product to yield S-folds, the authors of [17] argued that the theories on the resulting S-folds should have the scaling dimensions $k \Delta_{7}$ and that these correspond to a phase rotation of supercharges. They deduced from these that $k \Delta_{7}=2,3,4,6$, and consequently, they found that the S-folds that are compatible with $\mathbb{Z}_{k}$-actions are as follows [17]: $\mathbb{Z}_{2}$ S-folds with type $H_{2}, D_{4}, E_{6}$ 7-branes; ${ }^{11} \mathbb{Z}_{3}$ S-folds with type $H_{1}, D_{4}$ 7-branes; and $\mathbb{Z}_{4}$ S-folds with type $H_{2}$ 7-brane. The axio-dilaton has a constant value over the base space, and the value depends on the value of $k \Delta_{7}$ [17].

We remark here that the types of 7-branes for S-folds mentioned previously are the types before the quotient actions of $\mathbb{Z}_{k}$. The authors of [17] computed the $\mathbb{Z}_{k}$-quotients of the corresponding singular fiber types by analyzing the $\mathbb{Z}_{k}$-actions on the Weierstrass equations directly.

However, there is another method to compute the quotient fiber types, which we discuss here. The complex structure of a singular fiber is invariant under the action of the finite cyclic group $\mathbb{Z}_{k}$. Alternatively, the j-invariant of a singular fiber is unchanged under the quotient action of $\mathbb{Z}_{k}$. Utilizing this mathematical fact, one can deduce the singular fiber types as a result of the quotient action of $\mathbb{Z}_{k}$ groups. The types of singular fibers of elliptic

\footnotetext{
${ }^{11}$ Here $H_{l}, l=1,2$, represents the Argyres-Douglas theories [4-6] that arise from an $\mathrm{SU}(2)$ theory possessing $l+1$ flavors.
} 


\begin{tabular}{|c|c|c|c|c|c|}
\hline brane type & J-invariant & Monodromy & Order of Monodromy & \# of 7-branes & singularity \\
\hline$I_{0}^{*}$ & regular & $-\left(\begin{array}{ll}1 & 0 \\
0 & 1\end{array}\right)$ & 2 & 6 & $D_{4}$ \\
\hline$I_{b}$ & $\infty$ & $\left(\begin{array}{ll}1 & b \\
0 & 1\end{array}\right)$ & infinite & $b$ & $A_{b-1}$ \\
\hline$I_{b}^{*}$ & $\infty$ & $-\left(\begin{array}{ll}1 & b \\
0 & 1\end{array}\right)$ & infinite & $b+6$ & $D_{b+4}$ \\
\hline$I I$ & 0 & $\left(\begin{array}{cc}1 & 1 \\
-1 & 0\end{array}\right)$ & 6 & 2 & none. \\
\hline$I I^{*}$ & 0 & $\left(\begin{array}{cc}0 & -1 \\
1 & 1\end{array}\right)$ & 6 & 10 & $E_{8}$ \\
\hline$I I I$ & 1728 & $\left(\begin{array}{cc}0 & 1 \\
-1 & 0\end{array}\right)$ & 4 & 3 & $A_{1}$ \\
\hline$I I I^{*}$ & 1728 & $\left(\begin{array}{cc}0 & -1 \\
1 & 0\end{array}\right)$ & 4 & 9 & $E_{7}$ \\
\hline$I V$ & 0 & $\left(\begin{array}{cc}0 & 1 \\
-1 & -1\end{array}\right)$ & 3 & 4 & $A_{2}$ \\
\hline$I V^{*}$ & 0 & $\left(\begin{array}{cc}-1 & -1 \\
1 & 0\end{array}\right)$ & 3 & 8 & $E_{6}$ \\
\hline
\end{tabular}

Table 1. Types of the singular fibers of elliptic surfaces and their properties $[115,116]$ and wellknown brane interpretations. "Regular" for j-invariant of type $I_{0}^{*}$ indicates the fact that j-invariant of type $I_{0}^{*}$ fiber can take any finite value in $\mathbb{C}$.

surfaces, including their j-invariants, were classified by Kodaira in [115, 116]. Kodaira's classification of the types of singular fibers ${ }^{12}$ for elliptic surfaces is presented in table 1.

Because there are $\mathbb{Z}_{2}$ and $\mathbb{Z}_{4}$ S-folds with type $H_{2}$ 7-branes according to the results of [17], $\mathbb{Z}_{2^{-}}$and $\mathbb{Z}_{4}$-quotients of type $I V$ fibers can be considered. Type $I V$ fibers have j-invariant 0 according to table 1 ; therefore, $\mathbb{Z}_{2^{-}}$and $\mathbb{Z}_{4}$-quotients of this fiber type must have j-invariant 0 . The order of the $A_{2}$ singularity corresponding to type $I V$ fibers is 4 , and the $\mathbb{Z}_{2}$-action doubles this order, so the resulting fiber type has a corresponding singularity type of order 8 . Thus, we uniquely determine that the resulting fiber type is $I V^{*}$. This is consistent with the result given in [17]. A similar reasoning applied to the $\mathbb{Z}_{4}$-quotient appears to show that the resulting fiber type has order 16 , and this appears to be a bad singularity. In fact, this is not the case. To see this, consider the following reparameterization of the Weierstrass coefficients:

$$
f \rightarrow z^{2} \cdot f, \quad g \rightarrow z^{3} \cdot g
$$

Because the discriminant is given by $\Delta \sim 4 f^{3}+27 g^{2}$, this reparameterization reduces the order of the singularity by six, and the actual order of singularity is 10 . Therefore, the $\mathbb{Z}_{4}$-quotient of a type $I V$ fiber has a j-invariant whose corresponding singularity has order

\footnotetext{
${ }^{12}[117,118]$ discussed techniques to determine the types of the singular fibers of an elliptic fibration.
} 


\begin{tabular}{|c|c|}
\hline S-fold quotient of singular fiber & rank-one $4 \mathrm{~d} N=2$ SCFTs \\
\hline$I V^{*}, \mathbb{Z}_{2}$ & {$\left[I I^{*}, C_{5}\right]$} \\
$I_{0}^{*}, \mathbb{Z}_{2}$ & {$\left[I I I^{*}, C_{3} C_{1}\right]$} \\
$I_{0}^{*}, \mathbb{Z}_{3}$ & {$\left[I I^{*}, A_{3} \rtimes \mathbb{Z}_{2}\right]$} \\
$I V, \mathbb{Z}_{2}$ & {$\left[I V^{*}, C_{2} U_{1}\right]$} \\
$I V, \mathbb{Z}_{4}$ & {$\left[I I^{*}, A_{2} \rtimes \mathbb{Z}_{2}\right]$} \\
$I I I, \mathbb{Z}_{3}$ & {$\left[I I I^{*}, A_{1} U_{1} \rtimes \mathbb{Z}_{2}\right]$} \\
\hline
\end{tabular}

Table 2. Rank-one $4 \mathrm{~d} N=2$ SCFTs on S-folds with discrete torsion corresponding to quotients of Kodaira fibers [17] are listed. Items in the left column represent $\mathbb{Z}_{n}$ S-fold quotients of Kodaira fibers. For example, $I V^{*}, \mathbb{Z}_{2}$ indicates $\mathbb{Z}_{2}$ quotient of type $I V^{*}$ Kodaira fiber. The first entries in the square brackets in the right column represent the Kodaira fiber types corresponding to the Coulomb-branch singularities. The groups appearing as the second entries in the square brackets in the right column represent the flavor symmetries of the probe D3-branes in the resulting rank-one $4 \mathrm{~d} N=2$ SCFTs. We follow the notational convention utilized in [14].

10 , and this is uniquely determined to be a type $I I^{*}$ fiber. This is also consistent with the result in [17].

A similar reasoning applies to the $\mathbb{Z}_{k}$-quotient of other fiber types. The results of the quotient fiber types obtained in [17] are perfectly consistent with the results deduced via the method described herein.

S-fold quotient constrains the deformation parameters of the Weierstrass equation of an elliptic fibration [17, 22], and the mass deformation parameters form the Casimir invariants of the resulting flavor symmetry. The flavor symmetries of $4 \mathrm{~d} N=2$ SCFTs on S-folds were discussed in $[17,21,22]$. We list the rank-one $4 \mathrm{~d} N=2 \mathrm{SCFTs}$ on S-folds with discrete torsion and their flavor symmetries deduced in $[17]^{13}$ in table 2.

\section{$2.24 \mathrm{~d} N=1$ SCFTs as deformations of $4 \mathrm{~d} N=2$ SCFTs on S-folds, T-branes in $4 \mathrm{~d} N=1 \mathrm{~S}$-fold and brane monodromy}

One of the aims of this note is to analyze the $4 \mathrm{~d} N=1$ SCFTs obtained as deformations of the $4 \mathrm{~d} N=2$ SCFTs on S-folds [17] that we reviewed previously. We require an operation that breaks $N=2$ supersymmetry down to $N=1$; we utilize an operation of tilting a stack of 7-branes, as described in [45, 46]. This operation corresponds to the Higgs field $\phi$ on the 7-branes stack acquiring a non-zero position-dependent vev [45]. Then, tilting the stack of 7 -branes adds a correction to the superpotential as $\operatorname{Tr}_{G}\left(\phi\left(Z_{1}, Z_{2}\right) \cdot \mathcal{O}\right)$ [45]. $\mathcal{O}$ denotes dimension-two operators in the D3-brane probe theory transforming in the adjoint representation of $G$, where $G$ represents the flavor symmetry group [45]. When $\phi$ and $\phi^{\dagger}$ do not commute, the $N=2$ supersymmetry is broken down to $N=1$ [45]. $N=1$ deformation by tilting the stack of 7-branes corresponds to the superpotential deformation $\delta W=\operatorname{Tr}_{G}\left(\phi\left(Z_{1}, Z_{2}\right) \cdot \mathcal{O}\right)$ as discussed in $[45,46]$.

\footnotetext{
${ }^{13}$ Rank-one $4 \mathrm{~d} N=2$ SCFTs on S-folds without discrete torsion and their flavor symmetries can be found in [22].
} 
The adjoint-valued vev that $\phi$ takes, in the situation $\left[\phi, \phi^{\dagger}\right] \neq 0$, yields room to consider the T-brane structure [35]. When the stack of $n 7$-branes is located at $z_{1}=0$, the T-brane is generally given by the following spectral equation [35]:

$$
z_{1}^{n}+b_{2} z_{1}^{n-2}+b_{3} z_{1}^{n-3}+\ldots+b_{n}=0
$$

where $z_{1}$ denotes the spectral parameter, and $b_{2}, \ldots, b_{n}$ are polynomials in the variables of $z_{2}$ and $z_{3}$. Equation (2.7) can be seen as the spectral equation for $\phi$.

A T-brane is a structure associated with the coincident 7-branes that the Weierstrass equation cannot capture [39]. This physical degree of freedom in string theory is not fully determined by the defining equation of the geometry and still receives constraints from the geometry when an S-fold is considered. In other words, the structure of the T-brane must be compatible with the quotient action of the $\mathbb{Z}_{k}$ groups. We now explain this in more detail.

The $\mathbb{Z}_{k}$ quotient of $K 3^{\circ} \times \mathbb{C}^{2}, k=2,3,4$, was used to construct S-folds in [17]. $K 3^{\circ}$ denotes an open patch on the K3 surface, as previously mentioned. We focus on these S-folds because the $4 \mathrm{~d} N=1$ theories analyzed here are obtained as deformations of the $4 \mathrm{~d} N=2$ SCFTs constructed in [17].

The base of $K 3^{\circ}$ as an elliptic fibration is isomorphic to $\mathbb{C}$, and the coordinate of the base is denoted by $z_{1}$. The coordinates of $\mathbb{C}^{2}$ in the product $K 3^{\circ} \times \mathbb{C}^{2}$ are denoted by $z_{2}, z_{3}$. $\mathbb{Z}_{k}$ action are given by (2.4) [17], as mentioned in section 2.1, where phases $\phi_{2}, \phi_{3}$ satisfy the relation (2.5). The product $z_{2} z_{3}$ is invariant under $\mathbb{Z}_{k}$ action (2.4). $z_{1}$ is identified with $e^{\frac{2 \pi i}{k}} z_{1}$ under the quotient action.

Note here that when S-fold construction is considered, geometric conditions are imposed on the equation (2.7). For a $\mathbb{Z}_{k}$ S-fold, the coordinate $z$ satisfies the equivalence relation

$$
z_{1} \sim e^{\frac{2 \pi i}{k}} z_{1}
$$

For simplicity, we focus on the situations where the coefficients $b_{i}, i=2, \ldots, n$, are polynomials that only depend on the product $z_{2} z_{3}$ as a variable. Namely, we only consider the cases in which they are given as

$$
b_{i}=\sum_{j} c_{i j}\left(z_{2} z_{3}\right)^{j}
$$

As stated previously, the product $z_{2} z_{3}$ is invariant under $\mathbb{Z}_{k}$ action (2.4). Under this assumption, conditions are imposed only on powers of $z_{1}$ in the spectral equation (2.7) to make it compatible with the $\mathbb{Z}_{k}$ action. For simplicity of the argument, we focus on this situation and proceed.

The T-brane is then given by the spectral equation of the following form:

$$
F(z)=z_{1}^{n}+b_{2}\left(z_{2} z_{3}\right) z_{1}^{n-2}+b_{3}\left(z_{2} z_{3}\right) z_{1}^{n-3}+\ldots+b_{n}\left(z_{2} z_{3}\right)=0,
$$

where $b_{i}\left(z_{2} z_{3}\right), i=2, \ldots, n$, are polynomials in $z_{2} z_{3}$. The form of the T-brane must be invariant under $\mathbb{Z}_{k}$ action (2.4). Because the coefficients $b_{i}\left(z_{2} z_{3}\right), i=2, \ldots, n$ are invariant 
under $\mathbb{Z}_{k}$ action (2.4), this is equivalent to the requirement that $F(z)$ satisfies the following relation:

$$
F\left(e^{\frac{2 \pi i}{k}} \cdot z_{1}\right)=c F\left(z_{1}\right) .
$$

Here, $c$ denotes a non-zero constant number. When this condition is satisfied, under the quotient action of $\mathbb{Z}_{k}$, the equation $F(z)=0$ is invariant; the form of the T-brane is unchanged under the quotient action.

The condition (2.11) clearly constrains the form of the polynomial $F(z)$ in the spectral equation. The physical consequence of this ${ }^{14}$ is as follows. Because the Galois group of the polynomial $F(z)$ yields brane monodromy [35, 46], the S-fold compatibility condition (2.11) constrains the brane monodromy. When conditions are imposed on the coefficients of the polynomial $F(z)$, the associated Galois group generally becomes smaller than the symmetric group $S_{n}$. As a result, the brane monodromy group becomes smaller owing to the compatibility condition (2.11). Although the T-brane is a structure associated with the coincident 7-branes that the Weierstrass equation cannot fully capture, its structure still receives restrictions from the geometry, as we have shown. Compatibility with the $\mathbb{Z}_{k}$ orbifold actions physically constrains the structures of T-branes and brane monodromies.

As an example, we consider the T-brane structure in $4 \mathrm{~d}$ theory on an S-fold built from an open patch in K3 surface with an $A_{2}$ singularity corresponding to type IV fiber.

For this case, 7-branes in the open patch of the K3 surface have type $H_{2}$, and the type- $H_{2}$ stack consists of four 7 -branes. $\mathbb{Z}_{2}$ action acted on the open patch $\mathrm{K} 3^{\circ}$ times $\mathbb{C}^{2}$ yields $\mathbb{Z}_{2}$ S-fold. As we mentioned previously, the theory on probe D3-brane inserted in the resulting S-fold yields a $4 \mathrm{~d} N=2 \mathrm{SCFT},{ }^{15}$ and considering T-brane structure breaks half of $N=2$ supersymmetry which yields $4 \mathrm{~d} N=1$ theory. The spectral equation of the T-brane in the resulting $4 \mathrm{~d} N=1$ theory is given by degree-four characteristic polynomial. The spectral equation must satisfy the relation (2.11) under the action of $\mathbb{Z}_{2}$ group.

A T-brane given by the spectral equation of the following form is invariant under the $\mathbb{Z}_{2}$-action:

$$
F(z)=z_{1}^{4}+a z_{1}^{2}+b=0 .
$$

$a, b$ are polynomials in $z_{2} z_{3}$ as we previously mentioned. The equation (2.12) satisfies the relation (2.11). A computation shows that the Galois group of the polynomial (2.12) is isomorphic to $D_{8}$, the dihedral group of order 8 . Therefore, the 7-brane monodromy group for the $4 \mathrm{~d} N=1$ theory obtained from the $\mathbb{Z}_{2}$ S-fold is $D_{8}$.

Utilizing an open patch in $\mathrm{K} 3$ surface with an $A_{2}$ singularity corresponding to a type IV fiber, one can also construct a $\mathbb{Z}_{4}$ S-fold. For this construction, a T-brane described by the following equation is invariant under the $\mathbb{Z}_{4}$ action:

$$
F(z)=z_{1}^{4}+a=0 .
$$

\footnotetext{
${ }^{14}$ Possibilities that the coefficients $b_{i}$ take forms different from (2.9) are not excluded. The constraints imposed on the spectral equation by the compatibility with $\mathbb{Z}_{k}$ action (2.4), however, do not essentially change for such possibilities, and we expect that a physical consequence similar to that we discuss here applies to the possible situations wherein the coefficients $b_{i}$ take forms different from (2.9) in the spectral equation.

${ }^{15}$ This theory flows to a $4 \mathrm{~d}$ theory with $N=4$ supersymmetry, instead of $N=3$ theory [17, 24].
} 
The Galois group of this polynomial is isomorphic to $\mathbb{Z}_{4}$, and the 7-brane monodromy group for the $4 \mathrm{~d} N=1$ theory obtained from the constructed $\mathbb{Z}_{4}$ S-fold is $\mathbb{Z}_{4}$.

We make a remark on the flavor symmetries of the probe D3-branes in $4 \mathrm{~d} N=1 \mathrm{SCFTs}$ obtained as deformations of the $4 \mathrm{~d} N=2$ SCFTs on S-folds by T-branes. Because the effect of considering T-branes corresponds to tilting the stack of 7 -branes, we expect that the resulting flavor symmetry algebra of the probe D3-brane in $4 \mathrm{~d} N=1$ SCFT obtained as a deformation of a $4 \mathrm{~d} N=2 \mathrm{SCFT}$ on an S-fold by T-branes is a subalgebra of the flavor symmetry algebra of that $4 \mathrm{~d} N=2 \mathrm{SCFT}$. This argument suggests that the flavor symmetry algebras of the $4 \mathrm{~d} N=1$ SCFTs obtained as deformations of the $4 \mathrm{~d} N=2$ SCFTs on S-folds by T-branes are given as subalgebras of the flavor symmetry algebras of the $4 \mathrm{~d} N=2$ SCFTs deduced in $[12,17,21,22]$.

\section{Two $4 \mathrm{~d} N=1$ theories behaving identically in IR but behaving differently in UV}

We discussed structures of some $4 \mathrm{~d} N=1$ SCFTs probed by D3-branes in section 2. The D3-brane only probes a local small patch of the geometry, and theories behaving identically in IR do not necessarily behave identically in UV. In this section, we aim to provide two $4 \mathrm{~d} N=1$ theories that behave identically in IR but differently in UV by analyzing the geometry from a global perspective. When the global structures of the geometries are distinct, as theories flow to the UV regime, they become sensitive to the difference in the global structures of the geometries.

Our argument in this section applies to general geometries constructed as genus-one fibrations with multisections and their deformations into elliptic fibrations with multiple global sections. Our argument applies to $4 \mathrm{~d} N=1 \mathrm{SCFTs}$ constructed using S-folds, as discussed in section 2, as special cases of such geometries. As constructed in [63, 64], there are genus-one fibrations lacking a global section whose fibers have constant j-invariants 0 and 1728 throughout the base spaces (or equivalently, having complex structures $\tau=\exp \left(\frac{\pi i}{3}\right.$ ) and $\tau=i$ ). One can construct "closed" $\mathbb{Z}_{k}$ S-folds ${ }^{16}$ by acting $\mathbb{Z}_{k}$ groups on these genus-one fibrations. Because the D3-brane only probes a local open patch, theories on such closed S-folds in the IR limit reduce to $4 \mathrm{~d} N=1$ SCFTs on S-folds, as discussed in section 2.

We have stated the capacity to which our argument in this section applies. Before discussing the central point of our argument, we explain the physical meanings of multisection geometries and elliptic fibrations with multiple sections. It is known that a discrete gauge group arises on multisection geometry in F-theory [69]. A multisection is a multifold cover of the base space of a genus-one fibration. The times a multisection wraps around over the base is referred to as the "degree" of the multisection. When a multisection has degree $n$, it is concisely referred to as an " $n$-section." A discrete $\mathbb{Z}_{n}$ gauge group forms in F-theory on an $n$-section geometry [69]. The special case of $n=1$ corresponds to a global section, yielding a copy of the base space.

By tuning the coefficients of the defining equation for a genus-one fibration with a multisection to special values, a multisection splits into multiple global sections. The

\footnotetext{
16 "Closed" means that they have compact base spaces.
} 
number of independent global sections that an elliptic fibration possesses minus one yields the number of $\mathrm{U}(1) \mathrm{s}$ formed in F-theory on that elliptic fibration, as discussed in [82]. Therefore, when an $n$-section splits into $n$ global sections, a $\mathrm{U}(1)^{n-1}$ forms in F-theory. Tuning an $n$-section geometry to an elliptic fibration with $n$ sheets of global sections can be viewed as the reverse of the Higgsing process, wherein a model with $\mathrm{U}(1)^{n-1}$ breaks down into a mode with a $\mathbb{Z}_{n}$ gauge group [69].

It would be natural to expect that because the D3-brane only probes a small local patch of the geometry, it is insensitive to tuning of a genus-one fibration with an $n$-section into an elliptic fibration with $n$ global sections. We demonstrate that this expectation is true.

The key point to show this is that an $n$-section and $n$ separate global sections are locally indistinguishable. An $n$-section is an $n$-fold branched cover of the base space. In other words, an $n$-section is $n$ global sections combined along the branching loci. When the branching loci diminish and eventually disappear, the $n$-section splits into $n$ separate global sections. When one takes a small open patch such that it avoids any point in the branching loci, that local patch does not distinguish the $n$-section and $n$ separate global sections; the only difference is whether $n$ copies of the base space are combined along the branching loci or not, but that local patch does not pass any branched point, so they locally have no difference.

Now we tune a genus-one fibration with an $n$-section to an elliptic fibration with $n$ separate global sections while maintaining the type of the 7-branes stack at which the probe D3-brane is placed. The probe D3-brane is thus insensitive to this deformation in the geometry, as we have demonstrated.

In particular, this means that in the IR limit, the D3-brane does not probe the Higgsing process of $\mathrm{U}(1)^{n-1}$ breaking down into a $\mathbb{Z}_{n}$ group occurring through the global geometric deformation in the moduli. $\mathrm{U}(1)$ or $\mathbb{Z}_{n}$ groups arising from the global feature of the geometry in F-theory are not reflected in the $4 \mathrm{~d} N=1$ SCFT in IR.

For the two theories, realized as an F-theory on an $n$-section geometry and an F-theory on an elliptic fibration with $n$ global sections, the two theories on the D3-brane probes flow to the identical theory in IR. In UV, differences in the global geometric structures are probed and flow to distinct theories, reflecting the difference between $\mathrm{U}(1)^{n-1}$ and $\mathbb{Z}_{n}$ groups.

We would like to demonstrate that it is possible to deform a multisection geometry to an elliptic fibration with multiple global sections while maintaining the type of stack of the 7-branes at which the D3-brane probes. We show this for the case of a bisection geometry ${ }^{17}[64,68,69]$. A bisection geometry generally admits an expression as the double cover of a quartic polynomial, as discussed in [68, 69].

We particularly discuss bisection geometries given by equations of the following form, as studied in [64]:

$$
u^{2}=\prod_{i=1}^{4}\left(t-\alpha_{i}\right) x^{4}+\prod_{j=5}^{8}\left(t-\alpha_{j}\right),
$$

\footnotetext{
${ }^{17}$ Bisection refers to an $n$-section with $n=2$.
} 
where $t$ and $x$ represent inhomogeneous coordinates of $\mathbb{P}^{1}$, and each $\alpha_{i}, i=1, \ldots, 8$, is a point in $\mathbb{P}^{1}$. Thus, the equation (3.1) yields a double cover of $\mathbb{P}^{1} \times \mathbb{P}^{1}$ branched over a $(4,4)$ curve, which is a K3 surface [64]. A projection onto $\mathbb{P}^{1}$ in the product $\mathbb{P}^{1} \times \mathbb{P}^{1}$ yields a genus-one fibration, as discussed in [64]. We regard $t$ as the coordinate of the base $\mathbb{P}^{1}$ of the genus-one fibration. The K3 surface (3.1) is a bisection geometry [64]. The elliptic fibers of this fibration have a particular symmetry, which forces the complex structure of the fibers to be constant with $\tau=i,{ }^{18}$ throughout the base $\mathbb{P}^{1}$ [64]. This constrains the types of 7-branes so that the fiber types over the 7-branes can only be $I I I, I_{0}^{*}$, or $I I I^{*}[64]$.

We focus on the case of $\alpha_{5}=\alpha_{6}=0$ here. Then, the equation of the K3 surface (3.1) becomes

$$
u^{2}=\prod_{i=1}^{4}\left(t-\alpha_{i}\right) x^{4}+t^{2}\left(t-\alpha_{7}\right)\left(t-\alpha_{8}\right) .
$$

We only consider the cases where $\alpha_{i}, i=1,2,3,4$, are mutually distinct and $\alpha_{i}$, $i=1,2,3,4,7,8$ are non-zero. The discriminant of the K3 surface (3.2) is given by [64]

$$
\Delta \sim \prod_{i=1}^{4}\left(t-\alpha_{i}\right)^{3} \cdot t^{6}\left(t-\alpha_{7}\right)^{3}\left(t-\alpha_{8}\right)^{3} .
$$

7 -branes at the origin $t=0$ have type $D_{4}$, and each stack of 7 -branes at $t=\alpha_{i}, i=1,2,3,4$, has type $H_{1}$ [64]. 7-brane stacks at $t=\alpha_{7}, \alpha_{8}$ have type $H_{1}$ when $\alpha_{7}$ and $\alpha_{8}$ are not equal. At the limit at which $\alpha_{7}$ and $\alpha_{8}$ coincide, the 7-brane type at $t=\alpha_{7}$ is enhanced to $D_{4}$.

For the reader's convenience, we present the Jacobian fibration of the double cover (3.2). The Jacobian fibration admits transformation to the following Weierstrass form [119]:

$$
y^{2}=\frac{1}{4} x^{3}-\prod_{i=1}^{4}\left(t-\alpha_{i}\right) t^{2}\left(t-\alpha_{7}\right)\left(t-\alpha_{8}\right) x .
$$

We consider F-theory on a K3 surface (3.2) times a K3 surface. We consider the situation where the probe D3-brane is placed at the origin $t=0$. We consider tilting the stack of 7-branes at the origin $t=0$ to yield a $4 \mathrm{~d} N=1 \mathrm{SCFT}$, as discussed in section 2 . For general parameters $\alpha_{i}, i=1,2,3,4,7,8$, the K3 surface (3.2) has a bisection, but it does not have a global section, and a $\mathbb{Z}_{2}$ gauge group forms in F-theory. As we discussed previously, this is not probed by the D3-brane in IR.

One can tune the parameters so a bisection splits into two global sections. A bisection splits into global sections when either the coefficient of $x^{4}$ or constant term in the quartic polynomial becomes a perfect square [69]. This occurs when $\alpha_{7}$ and $\alpha_{8}$ become coincident in (3.2). Therefore, at the limit at which $\alpha_{7}$ and $\alpha_{8}$ coincide, the bisection splits into two global sections. Then, $\mathrm{U}(1)$, instead of the $\mathbb{Z}_{2}$ gauge group, forms in F-theory, but the theory on the D3-brane probe is insensitive to this change.

One can choose $\alpha_{7}$ and $\alpha_{8}$ far away from the origin so they do not lie in the local patch that the D3-brane probes. Tuning $\alpha_{8}$ such that it approaches $\alpha_{7}$ does not affect the singularity type at the origin $t=0$.

We particularly considered a bisection given by a specific form of an equation, but a similar reasoning applies to general bisection geometry.

\footnotetext{
${ }^{18}$ Equivalently, they have j-invariant 1728 .
} 


\section{Puzzle in the global structure of the gauge group}

When an elliptic fibration has a section, the set of sections is known to form a group, and this group is referred to as the "Mordell-Weil group." When the Mordell-Weil group of an elliptic fibration has a torsion part, the torsion part divides the global structure of the gauge group formed in F-theory on that elliptic fibration [111-113].

There are non-isomorphic elliptic K3 surfaces whose singular fiber types are identical and F-theory compactifications yield identical 7-brane types but their Mordell-Weil torsions are different. We explicitly give an example of this. We utilize extremal K3 surfaces. ${ }^{19}$ A complex elliptically fibered K3 surface $f: S \rightarrow \mathbb{P}^{1}$ with a global section is referred to as extremal when the Picard number of K3 surface $S$ is 20 and the Mordell-Weil group, $M W(S, f)$, is a finite group. Complex K3 surfaces with the Picard number of 20 are referred to as attractive K3 surfaces. ${ }^{20}$

It is a mathematical fact that the complex structures of the attractive K3 surfaces are labelled by triplets of integers [122, 123]. The complex structures, singularity types, and Mordell-Weil torsions of the K3 extremal fibrations were completely classified in [124]. A pair of non-isomorphic K3 surfaces whose singularity types are identical but Mordell-Weil torsions are different can be found in table 2 in [124]; numbers are assigned to the singularity types of the K3 extremal fibrations in this table. No. 276 in this table corresponds to the singularity type $E_{7} A_{9} A_{2}$. There are two K3 surfaces possessing this singularity type according to the table, and they have different complex structures, so they are nonisomorphic. F-theory on either of these two K3 surfaces times a K3 surface yields a 4d $N=2$ theory, and an $E_{7} \times \mathrm{SU}(10) \times \mathrm{SU}(3)$ gauge group forms in both compactifications. However, one of the extremal K3 surfaces has no Mordell-Weil torsion, while the other K3 surface has Mordell-Weil torsion isomorphic to $\mathbb{Z}_{2}$, so the gauge groups formed in the two theories are globally different. These theories ${ }^{21}$ yield different theories in UV, possessing distinct global structures of the gauge groups.

A natural question is whether the Mordell-Weil torsional group dividing the gauge group is reflected in the theory on the D3-brane probe, which probes only a local patch. Let us take a local patch of an elliptic fibration and place a probe D3-brane in the neighborhood of a stack of 7-branes. Locally, one cannot see the structures of global sections; thus, the Mordell-Weil group cannot be seen locally. Then, it is natural to consider that the MordellWeil torsions also cannot be locally seen. A consequence of this reasoning seems to be that the effect of Mordell-Weil torsion is not reflected in the $4 \mathrm{~d} N=1$ SCFT on the probe D3-brane in IR.

However, the 7-brane type is determined by the local information. In the language of F-theory, the 7-brane type is determined by the type of a singular fiber over the 7-branes,

\footnotetext{
${ }^{19}$ Discussions of extremal elliptic K3 surfaces in the context of string theory can be found, e.g., in $[63-67,120]$.

${ }^{20}$ We follow the convention of the term used in [121].

${ }^{21}$ We chose an example of extremal K3 fibrations, whose classification is known. However, the appearance of two non-isomorphic K3 surfaces whose singularity types are identical while having distinct Mordell-Weil torsions is not limited to extremal K3 surfaces, and such cases typically arise in the whole moduli of algebraic K3 surfaces.
} 
which is "vertical" information not relevant to the global feature of the base space. Because the Mordell-Weil torsional group acts on the gauge groups, the effect of this group might be reflected in the flavor symmetry group formed on the 7-branes that the D3-brane probes.

Because these two viewpoints provide opposing interpretations, one must choose one of the two interpretations. The question of which one gives the correct physical interpretation is left for future study.

\section{Concluding remarks and open problem}

In this note, we analyzed $4 \mathrm{~d} N=1$ SCFTs obtained as deformations of $4 \mathrm{~d} N=2$ SCFTs on S-folds by tilting 7-branes. We showed that T-branes receive constraints from the geometry when S-fold constructions are considered. Consequently, brane monodromies are also constrained by the geometric conditions, and we presented explicit examples of this.

We also discussed two $4 \mathrm{~d} N=1$ SCFTs behaving identically in IR but flowing to distinct theories in UV. To construct these theories, utilizing the global structure of genusone fibrations lacking a global section was useful. Our argument also showed that U(1) and discrete $\mathbb{Z}_{n}$ groups arising from the global feature of the geometry in F-theory are not reflected in $4 \mathrm{~d} N=1 \mathrm{SCFTs}$ on the probe D3-brane.

We encountered a dilemma when considering an effect of the global geometry on the theory living on the probe D3-brane, i.e., the action of the Mordell-Weil torsional group on the flavor symmetry group. Two opposing viewpoints seem possible for this problem, and which viewpoint yields a correct interpretation is left to be determined in future work.

We utilized the property that the D3-brane only probes a local patch of the geometry to deduce several physical consequences in sections 3 and 4. Strictly speaking, however, when the space possesses an S-fold singularity, not only the local patch of the geometry and the modes coming from the interaction of the D3-branes and the S-fold but also the structure of the S-fold can contribute to the $4 \mathrm{~d}$ physics, as explained in [24]. It might be interesting to investigate this subtle point, and this is left for future studies.

We remark that the conformal anomalies $a_{I R}$ and $c_{I R}$ for $4 \mathrm{~d} N=1$ SCFTs analyzed in this study can be computed. The value of the trial central charge, $a_{I R}(t)$, is expressed in terms of $a_{U V}, c_{U V}, k_{U V}$ and a parameter of the Jordan block structure pertaining to the superpotential deformation $\delta W=\operatorname{Tr}_{G}\left(\phi\left(Z_{1}, Z_{2}\right) \cdot \mathcal{O}\right)$ utilizing 't Hooft anomaly matching [46]. The conformal anomalies $a_{I R}$ and $c_{I R}$ are obtained by applying the method in [46], or the method in [125] with the geometric interpretation given in [126].

\section{Acknowledgments}

We would like to thank Yosuke Imamura, Shun'ya Mizoguchi and Shigeru Mukai for discussions. We are also grateful to the referee for improving this manuscript.

Open Access. This article is distributed under the terms of the Creative Commons Attribution License (CC-BY 4.0), which permits any use, distribution and reproduction in any medium, provided the original author(s) and source are credited. 


\section{References}

[1] N. Seiberg and E. Witten, Electric-magnetic duality, monopole condensation, and confinement in $N=2$ supersymmetric Yang-Mills theory, Nucl. Phys. B 426 (1994) 19 [Erratum ibid. 430 (1994) 485] [hep-th/9407087] [INSPIRE].

[2] N. Seiberg and E. Witten, Monopoles, duality and chiral symmetry breaking in $N=2$ supersymmetric QCD, Nucl. Phys. B 431 (1994) 484 [hep-th/9408099] [INSPIRE].

[3] P.C. Argyres and A.E. Faraggi, The vacuum structure and spectrum of $N=2$ supersymmetric $\mathrm{SU}(N)$ gauge theory, Phys. Rev. Lett. 74 (1995) 3931 [hep-th/9411057] [INSPIRE].

[4] P.C. Argyres and M.R. Douglas, New phenomena in SU(3) supersymmetric gauge theory, Nucl. Phys. B 448 (1995) 93 [hep-th/9505062] [INSPIRE].

[5] P.C. Argyres, M.R. Plesser, N. Seiberg and E. Witten, New $N=2$ superconformal field theories in four-dimensions, Nucl. Phys. B 461 (1996) 71 [hep-th/9511154] [InSPIRE].

[6] T. Eguchi, K. Hori, K. Ito and S.-K. Yang, Study of $N=2$ superconformal field theories in four-dimensions, Nucl. Phys. B 471 (1996) 430 [hep-th/9603002] [INSPIRE].

[7] J.A. Minahan and D. Nemeschansky, An $N=2$ superconformal fixed point with $E_{6}$ global symmetry, Nucl. Phys. B 482 (1996) 142 [hep-th/9608047] [INSPIRE].

[8] J.A. Minahan and D. Nemeschansky, Superconformal fixed points with E(n) global symmetry, Nucl. Phys. B 489 (1997) 24 [hep-th/9610076] [INSPIRE].

[9] P.C. Argyres and J.R. Wittig, Infinite coupling duals of $N=2$ gauge theories and new rank 1 superconformal field theories, JHEP 01 (2008) 074 [arXiv:0712.2028] [InSPIRE].

[10] D. Gaiotto, $N=2$ dualities, JHEP 08 (2012) 034 [arXiv:0904.2715] [INSPIRE].

[11] S. Cecotti, A. Neitzke and C. Vafa, R-Twisting and 4d/2d Correspondences, arXiv: 1006.3435 [INSPIRE].

[12] P. Argyres, M. Lotito, Y. Lü and M. Martone, Geometric constraints on the space of $\mathcal{N}=2$ SCFTs. Part I: physical constraints on relevant deformations, JHEP 02 (2018) 001 [arXiv: 1505.04814] [INSPIRE].

[13] D. Xie and S.-T. Yau, $4 d N=2$ SCFT and singularity theory Part I: Classification, arXiv: 1510.01324 [INSPIRE].

[14] P.C. Argyres and M. Martone, $4 d \mathcal{N}=2$ theories with disconnected gauge groups, JHEP 03 (2017) 145 [arXiv: 1611.08602] [INSPIRE].

[15] M. Caorsi and S. Cecotti, Geometric classification of $4 d \mathcal{N}=2$ SCFTs, JHEP 07 (2018) 138 [arXiv: 1801.04542] [INSPIRE].

[16] L. Borsten, M.J. Duff and A. Marrani, Twin conformal field theories, JHEP 03 (2019) 112 [arXiv:1812.11130] [INSPIRE].

[17] F. Apruzzi, S. Giacomelli and S. Schäfer-Nameki, $4 d \mathcal{N}=2$ S-folds, Phys. Rev. D 101 (2020) 106008 [arXiv:2001.00533] [INSPIRE].

[18] P. Argyres and M. Martone, Construction and classification of Coulomb branch geometries, arXiv: 2003.04954 [INSPIRE]. 
[19] Y.-H. He, E. Hirst and T. Peterken, Machine-learning dessins d'enfants: explorations via modular and Seiberg-Witten curves, J. Phys. A 54 (2021) 075401 [arXiv:2004.05218] [INSPIRE].

[20] A. Bourget, J.F. Grimminger, A. Hanany, M. Sperling, G. Zafrir and Z. Zhong, Magnetic quivers for rank 1 theories, JHEP 09 (2020) 189 [arXiv:2006.16994] [INSPIRE].

[21] S. Giacomelli, C. Meneghelli and W. Peelaers, New $\mathcal{N}=2$ superconformal field theories from $\mathcal{S}$-folds, JHEP 01 (2021) 022 [arXiv:2007.00647] [INSPIRE].

[22] J.J. Heckman, C. Lawrie, T.B. Rochais, H.Y. Zhang and G. Zoccarato, S-folds, string junctions, and $\mathcal{N}=2$ SCFTs, Phys. Rev. D 103 (2021) 086013 [arXiv:2009.10090] [INSPIRE].

[23] S. Giacomelli, M. Martone, Y. Tachikawa and G. Zafrir, More on $\mathcal{N}=2$ S-folds, JHEP 01 (2021) 054 [arXiv: 2010.03943] [inSPIRE].

[24] I. García-Etxebarria and D. Regalado, $\mathcal{N}=3$ four dimensional field theories, JHEP 03 (2016) 083 [arXiv: 1512.06434] [INSPIRE].

[25] O. Aharony and M. Evtikhiev, On four dimensional $N=3$ superconformal theories, JHEP 04 (2016) 040 [arXiv: 1512.03524] [INSPIRE].

[26] T. Nishinaka and Y. Tachikawa, On 4 d rank-one $\mathcal{N}=3$ superconformal field theories, JHEP 09 (2016) 116 [arXiv:1602.01503] [INSPIRE].

[27] O. Aharony and Y. Tachikawa, S-folds and $4 d N=3$ superconformal field theories, JHEP 06 (2016) 044 [arXiv: 1602.08638] [INSPIRE].

[28] Y. Imamura and S. Yokoyama, Superconformal index of $\mathcal{N}=3$ orientifold theories, J. Phys. A 49 (2016) 435401 [arXiv: 1603.00851] [InSPIRE].

[29] Y. Imamura, H. Kato and D. Yokoyama, Supersymmetry Enhancement and Junctions in S-folds, JHEP 10 (2016) 150 [arXiv:1606.07186] [INSPIRE].

[30] P. Agarwal and A. Amariti, Notes on S-folds and $\mathcal{N}=3$ theories, JHEP 09 (2016) 032 [arXiv: 1607.00313] [INSPIRE].

[31] M. Lemos, P. Liendo, C. Meneghelli and V. Mitev, Bootstrapping $\mathcal{N}=3$ superconformal theories, JHEP 04 (2017) 032 [arXiv: 1612.01536] [INSPIRE].

[32] J. van Muiden and A. Van Proeyen, The $\mathcal{N}=3$ Weyl multiplet in four dimensions, JHEP 01 (2019) 167 [arXiv: 1702.06442] [InSPIRE].

[33] T. Bourton, A. Pini and E. Pomoni, $4 d \mathcal{N}=3$ indices via discrete gauging, JHEP 10 (2018) 131 [arXiv: 1804.05396] [INSPIRE].

[34] R. Arai, S. Fujiwara and Y. Imamura, BPS Partition Functions for S-folds, JHEP 03 (2019) 172 [arXiv: 1901.00023] [InSPIRE].

[35] S. Cecotti, C. Cordova, J.J. Heckman and C. Vafa, T-Branes and Monodromy, JHEP 07 (2011) 030 [arXiv: 1010.5780] [INSPIRE].

[36] R. Donagi, S. Katz and E. Sharpe, Spectra of D-branes with Higgs vevs, Adv. Theor. Math. Phys. 8 (2004) 813 [hep-th/0309270] [INSPIRE].

[37] R. Donagi and M. Wijnholt, Gluing Branes, I, JHEP 05 (2013) 068 [arXiv:1104.2610] [INSPIRE]. 
[38] D.R. Morrison and W. Taylor, Matter and singularities, JHEP 01 (2012) 022 [arXiv:1106.3563] [INSPIRE].

[39] D.R. Morrison and W. Taylor, Toric bases for 6D F-theory models, Fortsch. Phys. 60 (2012) 1187 [arXiv: 1204.0283] [INSPIRE].

[40] L.B. Anderson, J.J. Heckman and S. Katz, T-Branes and Geometry, JHEP 05 (2014) 080 [arXiv:1310.1931] [INSPIRE].

[41] A. Collinucci and R. Savelli, T-branes as branes within branes, JHEP 09 (2015) 161 [arXiv:1410.4178] [INSPIRE].

[42] A. Collinucci and R. Savelli, F-theory on singular spaces, JHEP 09 (2015) 100 [arXiv: 1410.4867] [INSPIRE].

[43] M. Cicoli, F. Quevedo and R. Valandro, de Sitter from T-branes, JHEP 03 (2016) 141 [arXiv: 1512.04558] [INSPIRE].

[44] F. Hassler, J.J. Heckman, T.B. Rochais, T. Rudelius and H.Y. Zhang, T-Branes, String Junctions, and 6D SCFTs, Phys. Rev. D 101 (2020) 086018 [arXiv:1907.11230] [InSPIRE].

[45] J.J. Heckman and C. Vafa, An Exceptional Sector for F-theory GUTs, Phys. Rev. D 83 (2011) 026006 [arXiv: 1006.5459] [inSPIRE].

[46] J.J. Heckman, Y. Tachikawa, C. Vafa and B. Wecht, $N=1$ SCFTs from Brane Monodromy, JHEP 11 (2010) 132 [arXiv: 1009.0017] [INSPIRE].

[47] T. Banks, M.R. Douglas and N. Seiberg, Probing F-theory with branes, Phys. Lett. B 387 (1996) 278 [hep-th/9605199] [INSPIRE].

[48] M.R. Douglas, D.A. Lowe and J.H. Schwarz, Probing F-theory with multiple branes, Phys. Lett. B 394 (1997) 297 [hep-th/9612062] [INSPIRE].

[49] A. Fayyazuddin and M. Spalinski, Large $N$ superconformal gauge theories and supergravity orientifolds, Nucl. Phys. B 535 (1998) 219 [hep-th/9805096] [INSPIRE].

[50] O. Aharony, A. Fayyazuddin and J.M. Maldacena, The Large $N$ limit of $N=2, N=1$ field theories from three-branes in F-theory, JHEP 07 (1998) 013 [hep-th/9806159] [INSPIRE].

[51] S. Gukov and A. Kapustin, New $N=2$ superconformal field theories from $M / F$-theory orbifolds, Nucl. Phys. B 545 (1999) 283 [hep-th/9808175] [INSPIRE].

[52] I. Bah and B. Wecht, New $N=1$ Superconformal Field Theories In Four Dimensions, JHEP 07 (2013) 107 [arXiv:1111.3402] [InSPIRE].

[53] A. Gadde, K. Maruyoshi, Y. Tachikawa and W. Yan, New N=1 Dualities, JHEP 06 (2013) 056 [arXiv:1303.0836] [InSPIRE].

[54] J. McGrane and B. Wecht, Theories of class $\mathcal{S}$ and new $\mathcal{N}=1$ SCFTs, JHEP 06 (2015) 047 [arXiv: 1409.7668] [INSPIRE].

[55] K. Maruyoshi and J. Song, $\mathcal{N}=1$ deformations and $R G$ flows of $\mathcal{N}=2$ SCFTs, JHEP 02 (2017) 075 [arXiv: 1607.04281] [inSPIRE].

[56] T. Bourton, A. Pini and E. Pomoni, The Coulomb and Higgs branches of $\mathcal{N}=1$ theories of Class $\mathcal{S}_{k}$, JHEP 02 (2021) 137 [arXiv:2011.01587] [INSPIRE].

[57] H. Hayashi, T. Kawano, R. Tatar and T. Watari, Codimension-3 Singularities and Yukawa Couplings in F-theory, Nucl. Phys. B $\mathbf{8 2 3}$ (2009) 47 [arXiv:0901.4941] [InSPIRE]. 
[58] R. Donagi and M. Wijnholt, Higgs Bundles and UV Completion in F-theory, Commun. Math. Phys. 326 (2014) 287 [arXiv:0904.1218] [INSPIRE].

[59] V. Bouchard, J.J. Heckman, J. Seo and C. Vafa, F-theory and Neutrinos: Kaluza-Klein Dilution of Flavor Hierarchy, JHEP 01 (2010) 061 [arXiv:0904.1419] [INSPIRE].

[60] J.J. Heckman, A. Tavanfar and C. Vafa, The Point of $E_{8}$ in F-theory GUTs, JHEP 08 (2010) 040 [arXiv: 0906.0581] [INSPIRE].

[61] J. Marsano, N. Saulina and S. Schäfer-Nameki, Monodromies, Fluxes, and Compact Three-Generation F-theory GUTs, JHEP 08 (2009) 046 [arXiv: 0906. 4672] [INSPIRE].

[62] K. Dasgupta and S. Mukhi, F theory at constant coupling, Phys. Lett. B 385 (1996) 125 [hep-th/9606044] [INSPIRE].

[63] Y. Kimura, Gauge Groups and Matter Fields on Some Models of F-theory without Section, JHEP 03 (2016) 042 [arXiv:1511.06912] [INSPIRE].

[64] Y. Kimura, Gauge symmetries and matter fields in F-theory models without section compactifications on double cover and Fermat quartic K3 constructions times K3, Adv. Theor. Math. Phys. 21 (2017) 2087 [arXiv:1603.03212] [INSPIRE].

[65] Y. Kimura, Gauge groups and matter spectra in F-theory compactifications on genus-one fibered Calabi-Yau 4-folds without section: Hypersurface and double cover constructions, Adv. Theor. Math. Phys. 22 (2018) 1489 [arXiv: 1607.02978] [InSPIRE].

[66] Y. Kimura, Nongeometric heterotic strings and dual F-theory with enhanced gauge groups, JHEP 02 (2019) 036 [arXiv:1810.07657] [INSPIRE].

[67] Y. Kimura, Unbroken $E_{7} \times E_{7}$ nongeometric heterotic strings, stable degenerations and enhanced gauge groups in F-theory duals, arXiv:1902.00944 [INSPIRE].

[68] V. Braun and D.R. Morrison, F-theory on Genus-One Fibrations, JHEP 08 (2014) 132 [arXiv: 1401.7844] [INSPIRE].

[69] D.R. Morrison and W. Taylor, Sections, multisections, and U(1) fields in F-theory, arXiv: 1404.1527 [INSPIRE].

[70] L.B. Anderson, I. García-Etxebarria, T.W. Grimm and J. Keitel, Physics of F-theory compactifications without section, JHEP 12 (2014) 156 [arXiv:1406.5180] [INSPIRE].

[71] D. Klevers, D.K. Mayorga Pena, P.-K. Oehlmann, H. Piragua and J. Reuter, F-Theory on all Toric Hypersurface Fibrations and its Higgs Branches, JHEP 01 (2015) 142 [arXiv: 1408.4808] [INSPIRE].

[72] I. García-Etxebarria, T.W. Grimm and J. Keitel, Yukawas and discrete symmetries in F-theory compactifications without section, JHEP 11 (2014) 125 [arXiv:1408.6448] [INSPIRE].

[73] C. Mayrhofer, E. Palti, O. Till and T. Weigand, Discrete Gauge Symmetries by Higgsing in four-dimensional F-theory Compactifications, JHEP 12 (2014) 068 [arXiv:1408.6831] [INSPIRE].

[74] C. Mayrhofer, E. Palti, O. Till and T. Weigand, On Discrete Symmetries and Torsion Homology in F-theory, JHEP 06 (2015) 029 [arXiv: 1410.7814] [INSPIRE].

[75] V. Braun, T.W. Grimm and J. Keitel, Complete Intersection Fibers in F-theory, JHEP 03 (2015) 125 [arXiv: 1411.2615] [INSPIRE]. 
[76] M. Cvetič, R. Donagi, D. Klevers, H. Piragua and M. Poretschkin, F-theory vacua with $\mathbb{Z}_{3}$ gauge symmetry, Nucl. Phys. B 898 (2015) 736 [arXiv:1502.06953] [INSPIRE].

[77] L. Lin, C. Mayrhofer, O. Till and T. Weigand, Fluxes in F-theory Compactifications on Genus-One Fibrations, JHEP 01 (2016) 098 [arXiv: 1508.00162] [INSPIRE].

[78] Y. Kimura, Discrete Gauge Groups in F-theory Models on Genus-One Fibered Calabi-Yau 4-folds without Section, JHEP 04 (2017) 168 [arXiv:1608.07219] [INSPIRE].

[79] Y. Kimura, Discrete gauge groups in certain F-theory models in six dimensions, JHEP 07 (2019) 027 [arXiv: 1905.03775] [INSPIRE].

[80] Y. Kimura, A note on transition in discrete gauge groups in F-theory, Int. J. Mod. Phys. A 35 (2020) 2050144 [arXiv: 1907.13503] [INSPIRE].

[81] Y. Kimura, F-theory models with $\mathrm{U}(1) \times \mathbb{Z}_{2}, \mathbb{Z}_{4}$ and transitions in discrete gauge groups, JHEP 03 (2020) 153 [arXiv:1908.06621] [InSPIRE].

[82] D.R. Morrison and C. Vafa, Compactifications of F-theory on Calabi-Yau threefolds. 2., Nucl. Phys. B 476 (1996) 437 [hep-th/9603161] [inSPIRE].

[83] C. Vafa, Evidence for F-theory, Nucl. Phys. B 469 (1996) 403 [hep-th/9602022] [InSPIRE].

[84] D.R. Morrison and C. Vafa, Compactifications of F-theory on Calabi-Yau threefolds. 1, Nucl. Phys. B 473 (1996) 74 [hep-th/9602114] [InSPIRE].

[85] R. Donagi and M. Wijnholt, Model Building with F-theory, Adv. Theor. Math. Phys. 15 (2011) 1237 [arXiv: 0802 .2969] [inSPIRE].

[86] C. Beasley, J.J. Heckman and C. Vafa, GUTs and Exceptional Branes in F-theory - I, JHEP 01 (2009) 058 [arXiv:0802.3391] [InSPIRE].

[87] C. Beasley, J.J. Heckman and C. Vafa, GUTs and Exceptional Branes in F-theory - II: Experimental Predictions, JHEP 01 (2009) 059 [arXiv:0806.0102] [INSPIRE].

[88] R. Donagi and M. Wijnholt, Breaking GUT Groups in F-theory, Adv. Theor. Math. Phys. 15 (2011) 1523 [arXiv:0808.2223] [INSPIRE].

[89] D.R. Morrison and D.S. Park, F-Theory and the Mordell-Weil Group of Elliptically-Fibered Calabi-Yau Threefolds, JHEP 10 (2012) 128 [arXiv:1208.2695] [INSPIRE].

[90] C. Mayrhofer, E. Palti and T. Weigand, U(1) symmetries in F-theory GUTs with multiple sections, JHEP 03 (2013) 098 [arXiv:1211.6742] [INSPIRE].

[91] J. Borchmann, C. Mayrhofer, E. Palti and T. Weigand, Elliptic fibrations for $\mathrm{SU}(5) \times \mathrm{U}(1) \times \mathrm{U}(1)$ F-theory vacua, Phys. Rev. D 88 (2013) 046005 [arXiv:1303.5054] [INSPIRE].

[92] I. Antoniadis and G.K. Leontaris, F-GUTs with Mordell-Weil U(1)'s, Phys. Lett. B 735 (2014) 226 [arXiv: 1404.6720] [INSPIRE].

[93] A. Malmendier and D.R. Morrison, K3 surfaces, modular forms, and non-geometric heterotic compactifications, Lett. Math. Phys. 105 (2015) 1085 [arXiv:1406.4873] [INSPIRE].

[94] C. Lawrie, S. Schäfer-Nameki and J.-M. Wong, F-theory and All Things Rational: Surveying U(1) Symmetries with Rational Sections, JHEP 09 (2015) 144 [arXiv: 1504.05593] [INSPIRE]. 
[95] M. Cvetič, D. Klevers, H. Piragua and W. Taylor, General U(1) $\times$ U(1) F-theory compactifications and beyond: geometry of unHiggsings and novel matter structure, JHEP 11 (2015) 204 [arXiv: 1507.05954] [INSPIRE].

[96] D.R. Morrison, D.S. Park and W. Taylor, Non-Higgsable abelian gauge symmetry and F-theory on fiber products of rational elliptic surfaces, Adv. Theor. Math. Phys. 22 (2018) 177 [arXiv: 1610.06929$]$ [INSPIRE].

[97] Y. Kimura, F-theory models on K3 surfaces with various Mordell-Weil ranks constructions that use quadratic base change of rational elliptic surfaces, JHEP 05 (2018) 048 [arXiv: 1802.05195] [INSPIRE].

[98] S. Mizoguchi and T. Tani, Non-Cartan Mordell-Weil lattices of rational elliptic surfaces and heterotic/F-theory compactifications, JHEP 03 (2019) 121 [arXiv: 1808.08001] [INSPIRE].

[99] S.-J. Lee and T. Weigand, Swampland Bounds on the Abelian Gauge Sector, Phys. Rev. D 100 (2019) 026015 [arXiv: 1905.13213] [INSPIRE].

[100] Y. Kimura, $\frac{1}{2}$ Calabi-Yau 3-folds, Calabi-Yau 3-folds as double covers, and F-theory with $\mathrm{U}(1) s, J H E P 02$ (2020) 076 [arXiv: 1910.00008] [INSPIRE].

[101] C.F. Cota, A. Klemm and T. Schimannek, Topological strings on genus one fibered Calabi-Yau 3-folds and string dualities, JHEP 11 (2019) 170 [arXiv:1910.01988] [INSPIRE].

[102] L. Chabrol, F-theory and Heterotic Duality, Weierstrass Models from Wilson lines, Eur. Phys. J. C 80 (2020) 944 [arXiv: 1910.12844] [INSPIRE].

[103] Y. Kimura, $\frac{1}{2}$ Calabi-Yau 4-folds and four-dimensional F-theory on Calabi-Yau 4-folds with U(1) factors, arXiv:1911.03960 [INSPIRE].

[104] S. Fukuchi, N. Kan, R. Kuramochi, S. Mizoguchi and H. Tashiro, More on a dessin on the base: Kodaira exceptional fibers and mutually (non-)local branes, Phys. Lett. B 803 (2020) 135333 [arXiv: 1912.02974] [INSPIRE].

[105] N. Kan, S. Mizoguchi and T. Tani, Half-hypermultiplets and incomplete/complete resolutions in F-theory, JHEP 08 (2020) 063 [arXiv: 2003.05563] [INSPIRE].

[106] A. Karozas, G.K. Leontaris, I. Tavellaris and N.D. Vlachos, On the LHC signatures of $\mathrm{SU}(5) \times \mathrm{U}(1)^{\prime}$ F-theory motivated models, Eur. Phys. J. C 81 (2021) 35 [arXiv: 2007.05936] [INSPIRE].

[107] C. Angelantonj, Q. Bonnefoy, C. Condeescu and E. Dudas, String Defects, Supersymmetry and the Swampland, JHEP 11 (2020) 125 [arXiv:2007.12722] [INSPIRE].

[108] R. Kuramochi, S. Mizoguchi and T. Tani, Magic square and half-hypermultiplets in F-theory, arXiv:2008.09272 [INSPIRE].

[109] Y.-H. He and A. Lukas, Machine Learning Calabi-Yau Four-folds, Phys. Lett. B 815 (2021) 136139 [arXiv: 2009.02544] [INSPIRE].

[110] A. Clingher and A. Malmendier, On K3 surfaces of Picard rank 14, arXiv:2009.09635 [INSPIRE].

[111] P.S. Aspinwall and M. Gross, The $\mathrm{SO}(32)$ heterotic string on a K3 surface, Phys. Lett. B 387 (1996) 735 [hep-th/9605131] [INSPIRE].

[112] P.S. Aspinwall and D.R. Morrison, Nonsimply connected gauge groups and rational points on elliptic curves, JHEP 07 (1998) 012 [hep-th/9805206] [INSPIRE]. 
[113] C. Mayrhofer, D.R. Morrison, O. Till and T. Weigand, Mordell-Weil Torsion and the Global Structure of Gauge Groups in F-theory, JHEP 10 (2014) 016 [arXiv:1405.3656] [INSPIRE].

[114] J.W.S. Cassels, Lectures on Elliptic Curves, London Math. Society Student Texts 24, Cambridge University Press, Cambridge U.K. (1991) [DOI].

[115] K. Kodaira, On compact analytic surfaces II, Annals Math. 77 (1963), 563.

[116] K. Kodaira, On compact analytic surfaces III, Annals Math. 78 (1963), 1.

[117] A. Néron, Modèles minimaux des variétés abéliennes sur les corps locaux et globaux, Publ. Math., Inst. Hautes Étud. Sci. 21 (1964), 5.

[118] J. Tate, Algorithm for determining the type of a singular fiber in an elliptic pencil, in Modular Functions of One Variable IV, Springer, Berlin Germany (1975) [DOI].

[119] S. Mukai, An introduction to invariants and moduli, Cambridge University Press, U.K. (2003) [DOI].

[120] A. Font, B. Fraiman, M. Graña, C.A. Núñez and H.P. De Freitas, Exploring the landscape of heterotic strings on $T^{d}$, JHEP 10 (2020) 194 [arXiv: 2007.10358] [INSPIRE].

[121] G.W. Moore, Strings and Arithmetic, in Les Houches School of Physics: Frontiers in Number Theory, Physics and Geometry, (2004) DOI [hep-th/0401049] [INSPIRE].

[122] I.I. Piatetski-Shapiro and I.R. Shafarevich, A Torelli theorem for algebraic surfaces of type K3, Izv. Akad. Nauk SSSR Ser. Mat. 35 (1971), 530.

[123] T. Shioda and H. Inose, On Singular K3 surfaces, in W.L. Jr. Baily and T. Shioda (eds.), Complex analysis and algebraic geometry, Iwanami Shoten, Tokyo Japan (1977) [DOI].

[124] I. Shimada and D.-Q. Zhang, Classification of extremal elliptic K3 surfaces and fundamental groups of open K3 surfaces, Nagoya Math. J. 161 (2001), 23 [math/0007171].

[125] F. Apruzzi, F. Hassler, J.J. Heckman and T.B. Rochais, Nilpotent Networks and $4 D R G$ Flows, JHEP 05 (2019) 074 [arXiv: 1808.10439] [INSPIRE].

[126] F. Carta, S. Giacomelli and R. Savelli, SUSY enhancement from T-branes, JHEP 12 (2018) 127 [arXiv: 1809.04906] [INSPIRE]. 